\title{
InP-Based Spotsize Converter for Integration with Switching Devices
}

\author{
J. Stulemeijer, A. F. Bakker, I. Moerman, Member, IEEE, F. H. Groen, and M. K. Smit, Associate Member, IEEE
}

\begin{abstract}
We have designed and fabricated an InGaAsP-InP based spotsize converter (SSC), which is compatible with the waveguide structure used in monolithic integrated ADM's and OXC's. In a first experiement, a total coupling loss to a cleaved fiber of 4.2 dB for TE and 3.9 dB for TM was realized with a 1.5mm-long SSC. The 1-dB alignment tolerances improved from 0.75 $\mu \mathrm{m}$ to 2.4 and $2.2 \mu \mathrm{m}$ for the horizontal and vertical direction, respectively.
\end{abstract}

Index Terms - Integrated optics, optical communication, optical fiber connecting, optical waveguides, wavelength-division multiplexing.

\section{INTRODUCTION}

$\mathbf{E}$ FFICIENT fiber-chip coupling is of key importance to the application of photonic integrated circuits (PIC's). The usual semiconductor waveguide, applied in our lab [1], is shown in Fig. 1. The spotsize is on the order of $1 \times$ $0.5 \mu \mathrm{m}^{2}$. The small spotsize allows for the miniaturization of passive waveguide structures and for the integration with structures like lasers and optical phase shifters, but it poses severe problems for coupling light into a fiber.

When using a tapered fiber to couple light directly into the waveguide on the chip, the coupling loss is $5 \mathrm{~dB}$ and the alignment tolerances for $1-\mathrm{dB}$ extra loss are $0.75 \mu \mathrm{m}$. Spotsize converters (SSC's) transform the waveguide on the chip mode into a mode which is better adapted to a singlemode fiber (SMF). Using a SSC, the alignment tolerances are similar to the situation of coupling light from one SMF to another SMF, which gives 1-dB extra loss for a displacement of $2.4 \mu \mathrm{m}$. For a review on SSC's, see [2]. A state of the art SSC as made by ETH Zurich, based on a combination of diffusion-limited etch and diffusion-limited growth, obtained a polarization independent coupling loss of $1 \mathrm{~dB}$ for fiber-chip coupling, see [3]. Previously, we reported first results on an SSC, which is realized wit a single epitaxial growth step and is suitable for integration with WDM OXC's and ADM's, as published by our group in [4].

Manuscript received July 15, 1998; revised September 9, 1998

J. Stulemeijer and M. K. Smit are with Department of Information Technology and Systems, TTT Laboratory, Integrated Photonics Group, Delft University of Technology, 2600 GA Delft, The Netherlands.

A. F. Bakker is with BBV Software, 7521 PA Enschede, The Netherlands.

I. Moerman is with the Department of Information Technology, University of Gent-INTEC, B-9000 Gent, Belgium.

F. H. Groen is with Faculty of Applied Sciences Research Group for Optics, Delft University of Technology, 2600 GA Delft, The Netherlands.

Publisher Item Identifier S 1041-1135(99)00371-7.

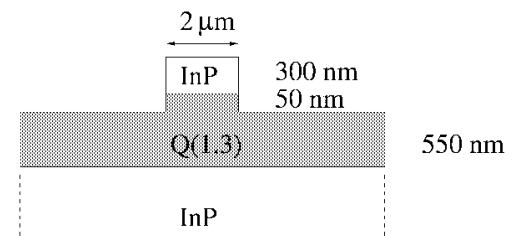

Fig. 1. Double-hetero ridge waveguide structure on our PIC's.

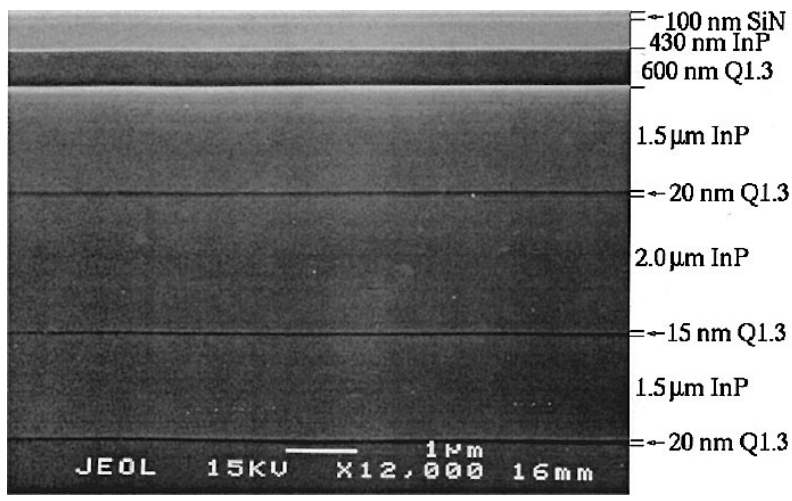

Fig. 2. Layerstack for SSC, which allows for the fabrication of both a high-contrast waveguide and a fiber-matched waveguide.

\section{DESIGN}

We have designed a layerstack (see Fig. 2) such that our normal compact waveguide and a large fiber matched waveguide can be made on the same substrate, as shown in Fig. 3. The large waveguide consists of three thin $Q(1.3)$ layers, which do not affect the properties of the compact waveguide. The structure is designed for use in the third window of optical communication at $1550 \mathrm{~nm}$.

The large waveguide gives a theoretical coupling loss of 0.5 $\mathrm{dB}$ to a SMF. Our design is very similar to the SSC reported in [4], with the difference that we use the InP-InGaAsP system instead of a doping profile to fabricate a fiber matched waveguide.

The lateral transition from the small spot waveguide to the large spot waveguide was realized by using a $120-\mu \mathrm{m}$-long parabolic taper which tapers the lateral width of the upper waveguide from 2 to $12 \mu \mathrm{m}$.

The transition in the transverse direction is more difficult. Here, we use a taper in the vertical direction, as shown in Fig. 4. In the direction from the chip to the fiber the height of 


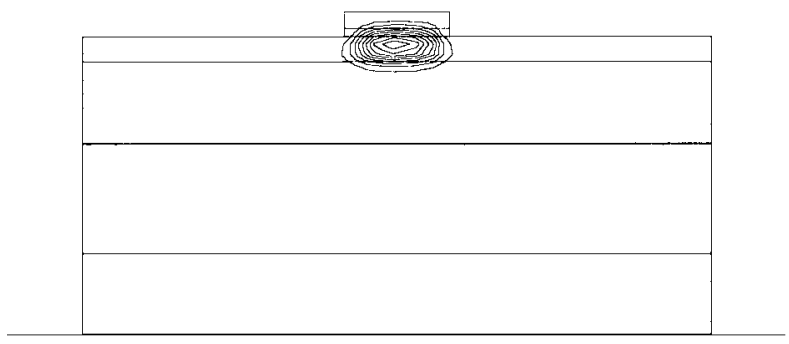

(a)

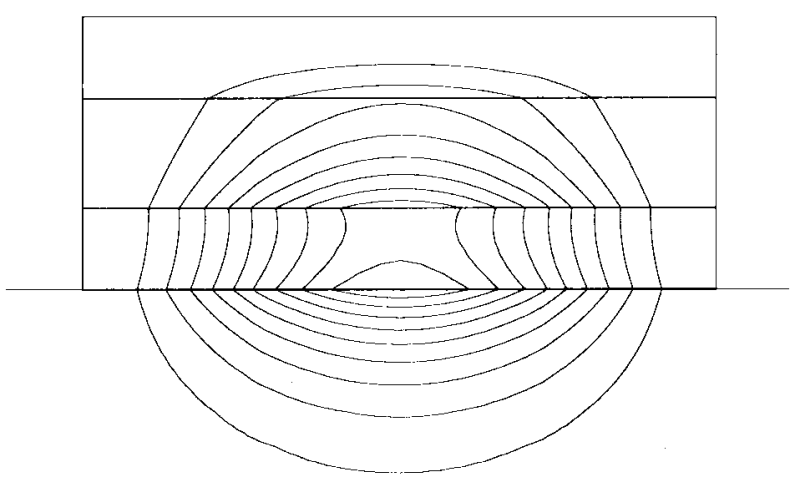

(b)

Fig. 3. On the left, the spot of the normal high-contrst waveguide is shown On the right, on the same scale as the left, the modal field in the fiber-matched waveguide is shown.

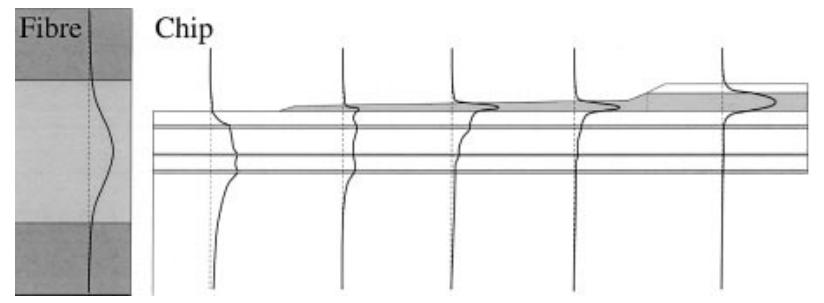

Fig. 4. Transformation of the modal field in a fiber to the modal field in the high-contrast waveguide on the chip.

the upper waveguide is tapered down to zero, thus the light is forced into the substrate. Notice that the profile (see Fig. 4) in the vertical direction is not linear. This profile was designed such that the taper angle is small around the film height where the light crosses over from the normal film layer to the three thin layers below. The height of the film at which the light crosses over depends on the polarization of the light.

The complete SSC is formed by putting the horizontal and vertical taper in series, see Fig. 5.

\section{FABRICATION}

The fabrication process is outlined in Fig. 6. First, the vertical taper is made.

This is done by using a diffusion limited etch based on $\mathrm{Br}_{2}: \mathrm{CH}_{3} \mathrm{OH} \mathrm{1:2000}$ at room temperature, see [5]. Due to the diffusion limited character of the etch the source of the reactant material at one point is restricted to a region with dimensions equal to the diffusion length. As a result the etch rate in a wide slit will be lower than the etch rate in a narrow

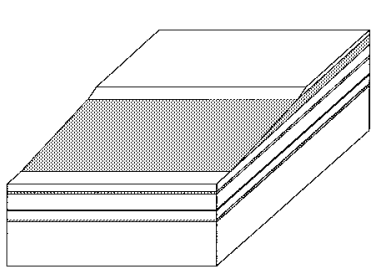

(a)

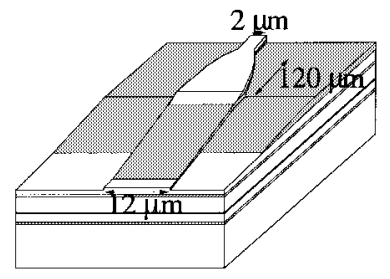

(b)

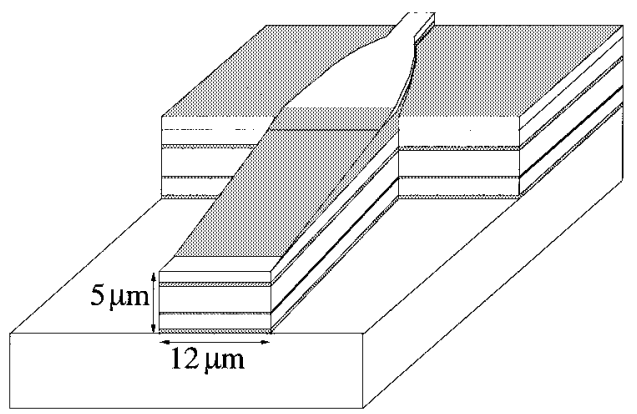

(c)

Fig. 5. Three steps in the fabrication of the SSC's.

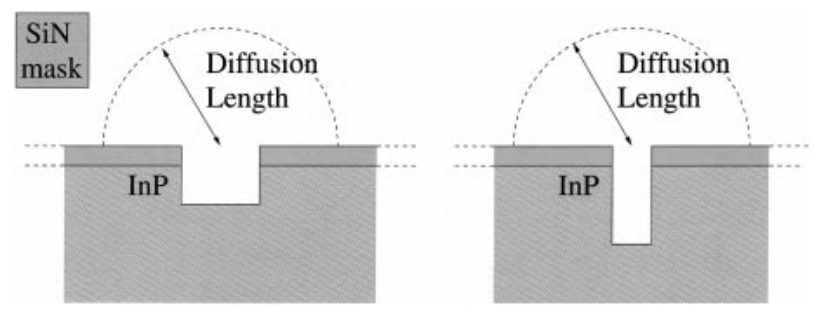

Fig. 6. Diffusion-limited etch. The same amount of etchant reacts with the surface through the slit, therefore, the etch rate increases with decreasing width of the slit.

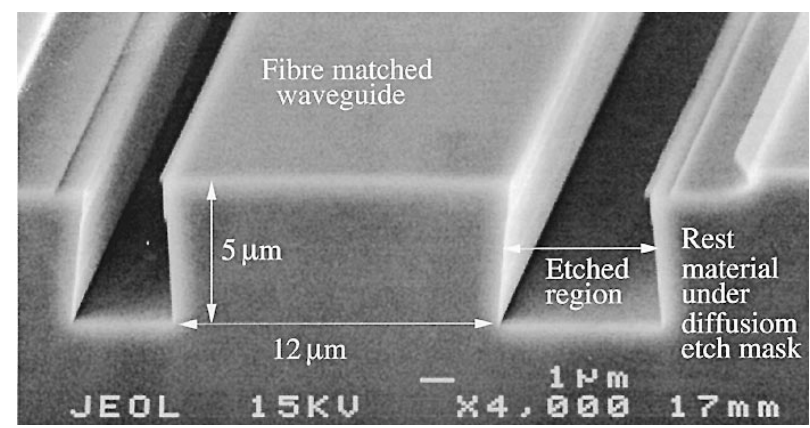

Fig. 7. SEM picture of the end face of an SSC.

slit, due to the depletion of the reactants. This is illustrated in Fig. 7.

So the height profile can be created by defining a slit of varying width on the surface of the chip, such that in the smallest opening of the slit the etchant will remove the top cladding plus the film layer. In the area that is completely left open, only a small amount of the cladding is etched away, see Fig. 8.

Next, the upper waveguide is etched using a $\mathrm{CH}_{4}: \mathrm{H}_{2} \mathrm{RIE}$ process. Finally, the fiber matched waveguide is etched using a series of selective wet chemical etches for InP and $Q(1.3)$. The end face of the resulting SSC is shown in Fig. 7. 


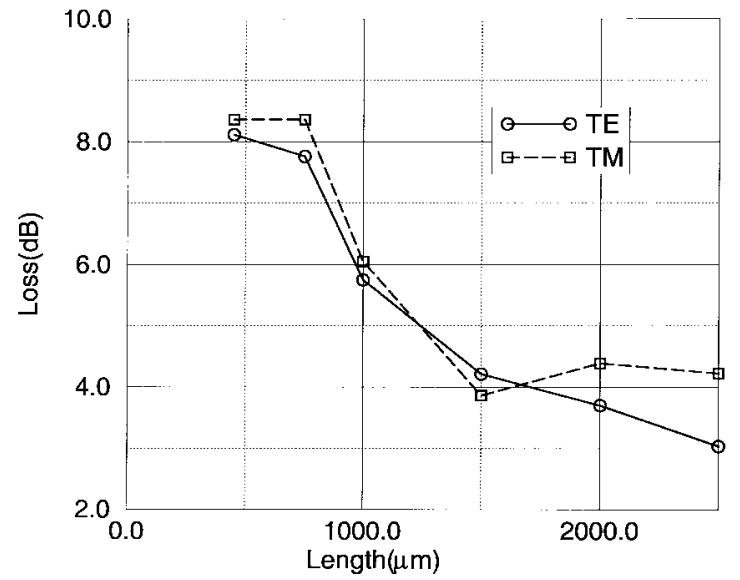

Fig. 8. Total losses of polarization independent SSC's.

\section{EXPERIMENTAL RESULTS}

The losses of the SSC's were measured by inserting a $2-\mu \mathrm{m}$-wide waveguide with SSC's on either end between two cleaved SMF's. Both the fibers and the chip are AR coated. The losses of a single SSC follow by measuring the total loss between two fibers, subtracting the losses of the $2-\mu \mathrm{m}$-wide straight waveguide $(0.7 \mathrm{~dB} / \mathrm{cm})$ and dividing the remainder by two. The losses for the $2-\mu \mathrm{m}$ waveguide are obtained by Fabry-Perot measurement before the AR coating was applied.

On the chip, six sets of five identical waveguides with SSC's were fabricated. The losses, coupling loss and modal conversion loss, are plotted in Fig. 8. The alignment tolerances for 1-dB extra loss were measured to be 2.6 and $2.2 \mu \mathrm{m}$ in the horizontal an vertical directions, respectively.

For a 1.5-mm-long SSC total losses of 4.2 and $3.9 \mathrm{~dB}$ were measured for TE and TM polarized light, respectively. Theoretically, $1.5 \mathrm{~dB}$ was expected, based on the modal conversion losses $(1 \mathrm{~dB})$ computed by BPM and coupling losses $(0.5 \mathrm{~dB})$ computed with a mode solver.

The discrepancy between theory and experiment can be attributed to two effects. Firstly the etched region besides the waveguide, which was designed at $6 \mu \mathrm{m}$, appeared to be much smaller due to some problems in the fabrication, see Fig. 7. For some SSC's, the etched region turned out to be as narrow as $2 \mu \mathrm{m}$. As a result part of the guided light leaks out of the wide waveguide. This is confirmed by a BPM simulation. Secondly, inhomogeneities (etch depth variations of $50 \mathrm{~nm}$ ) in the diffusion limited etch cause the vertical taper profile to not be optimal, which results in increased mode transformation losses. This also explains some of the fluctuating polarization dependent loss since the optimal vertical taper profile for TE is different from the optimal profile for TM.

With a wider etched region, larger than $4 \mu \mathrm{m}$, we expect to be able to reduce the total loss. This is confirmed by earlier experiments, see [4], in which the etched region beside the fiber matched ridge was sufficiently large. The main difference

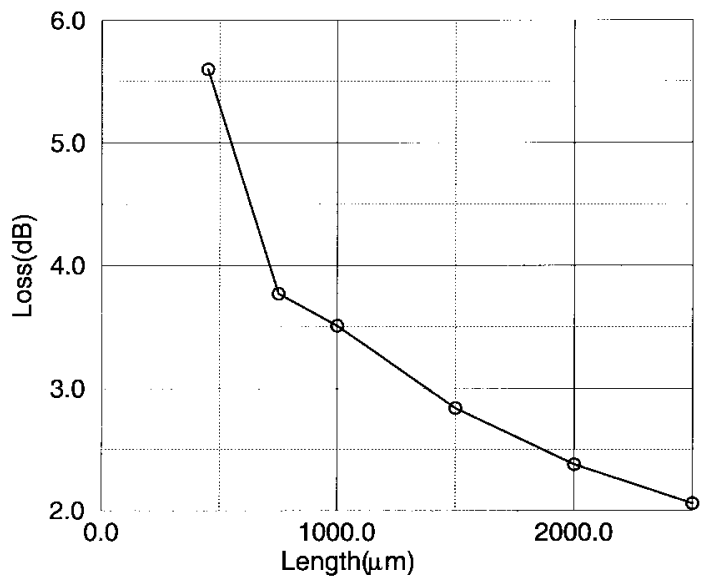

Fig. 9. Total losses of first SSC's for TE polarized light.

with the SSC described here is that we combined the vertical and the horizontal taper in the same region. This combination appeared to lead to increased losses for TM polarized light. The total losses for TE polarized light are plotted in Fig. 9 as a function of the length of the SSC. From this result we conclude that a 1-mm-long SSC with less than 3.5-dB loss is feasible in the present approach.

\section{CONCLUSION}

We realized a $1.5-\mathrm{mm}$-long SSC with losses of $4 \mathrm{~dB}$ and a polarization dependence of $0.3 \mathrm{~dB}$. It was shown that a SSC with 1-mm length and a loss below $3.5 \mathrm{~dB}$ for both polarizations, which is suitable for integration with optical ADM's and OXC's, is feasible. The alignment tolerances for $1-\mathrm{dB}$ extra loss are better than $2 \mu \mathrm{m}$ for both horizontal and vertical directions.

\section{REFERENCES}

[1] C. G. P. Herben, C. G. M. Vreeburg, D. H. P. Maat, X. J. M. Leijtens, Y. S. Oei, F. H. Groen, J. W. Pederson, P. Demeester, and M. K. Smit, "A compact integrated InP-based single-phasar optical crossconnect," IEEE Photon. Technol. Lett., vol. 10, pp. 678-680, May 1998.

[2] I. Moerman, P. P. Van Daele, and P. M. Demeester, "A review on fabrication technologies for the monolithic integration of tapers with III-V semiconductor devices," IEEE J. Select. Topics Quantum Electron., vol. 6, pp. 1308-1320, June 1998.

[3] T. Brenner, M. Bachmann, and H. Melchior, "Vertically tapered InGaAsP/InP waveguides for highly efficient coupling to flat-end singlemode fibers," Appl. Phys. Lett., vol. 65, pp. 798-800, Aug. 1994.

[4] G. Wegner, L. Stoll, B. Weiss, M. Schienle, R. Müller-Nawrath, S. Eichinger, J. Müller, B. Acklin, and G. Müller, "Design and fabrication of monolithic optical spot size transformers (MOST's) for highly efficient fiber-chip coupling," J. Lightwave Technol., vol. 12, pp. 1782-1790, Oct. 1994

[5] T. Brenner and H. Melchior, "Local etch-rate control of masked InP/InGaAsP by diffusion-limited etching," J. Electrochem. Soc., vol. 141, no. 7, pp. 1954-1956, 1994.

[6] J. Stulemeijer, A. F. Bakker, I. Moerman, F. H. Groen, and M. K. Smit, "Efficient InP-based integrable spot-size converter," in Int. Photonics Research Tech. Dig. (IPR'98), Opt. Soc. Amer., Mar./Apr. 1998, pp. 192-194. 